\title{
Prevalence of ocular involvement in pemphigus vulgaris patients in the dermatology department of Mayo Hospital Lahore
}

\begin{abstract}
Pemphigus vulgaris (PV) is a bullous disease. It has autoimmune etiology. It mainly affects skin along with mucous membranes. The frequency and pattern of involvement of eyes in this disease is not clearly defined till now. We decided to conduct a study of hundred patients with diagnosis of PV in a tertiary care hospital in Pakistan. The result showed a significant number of patients with eye involvement, 32 out of 100 cases in the indoor department of hospital i.e. (32\%) showed eye involvement in a variable range of severity and presentation. Most common symptom was conjunctivitis followed by palpebral conjunctival erosions. Discharge was present in few patients that was either watery or mucoid due to the secondary infection.
\end{abstract}

Volume 3 Issue 5 - 2019

\begin{abstract}
Ghazala Butt, M Arslan Ibrahim, Ahmad Raza, Uzair Wazir, Umara Siddique, Uzma Malik

Department of Dermatology, Mayo Hospital, Pakistan
\end{abstract}

Correspondence: Ghazala Butt, Assistant professor of Dermatology, Mayo Hospital, Pakistan,

Email ghazalakashmiri@gmail.com

Received: February 21, 2019 | Published: October 31, 2019

Keywords: pemphigus vulgaris, bullous disease, conjunctivitis, discharge

\section{Introduction}

Pemphigus vulgaris, bullous pemphigoid and other bullous disorders may have eye involvement in various range of severity. Patients of pemphigus vulgaris have skin and mucous membrane involvement. Acantholysis is present in suprabasilar layer of epithelium i.e. intraepithelial. On Biopsy, IgG can be confirmed by immunofloresecence and in circulation antiepithelial autoantibodies are found. There is a correlation with MHC II class genes. Majority of Ashkenazi Jews having a diagnosis of pemphigus vulgaris have DRB1 allelle. Pemphigus vulgaris is caused by auto-antibodies against desmoglein(a glycoprotein that is present in desmosomes ,tight junctions between epithelial cells). Desmoglein has also been found in the mucous membranes epithelial cell junction that describes the involvement of eye in pemphigus vulgaris.

The initial and common presentation is inflammation as seen in all autoimmune phenomena. As pemphigus vulgaris is an autoimmune and inflammatory disease, it responds well to immunosuppressive medication. Steroids are given intravenously/oral depending upon condition of patient and severity of the disease. There are various other immunosuppressive medicines e.g, azathioprine, cyclosporine, cyclophosphamide mycophenolate mofetil etc. Medical literature was sparse concerning ocular involvement in PV. In cooperation with the International Pemphigus Foundation a survey was undertaken to determine the prevalence and extent of ocular involvement in this rare disease.

\section{Methods}

After taking written informed consent hundred patients fulfilling the inclusion criteria were included in the study and were questioned and examined according to the International Pemphigus Questionnaire that appeared in the Foundation's website, discussion group and quarterly newsletter. A total of 158 responses were collected in 1999. Of these, 134 were from patients with PV and only those responses were considered. ${ }^{1-7}$

\section{Results}

This study included 100 pemphigus vulgaris patients who got admitted in the Indoor of Dermatology department, Mayo hospital during the year 2017 and 2018 upto month of May. The study was conducted based upon a questionnaire that included history of disease, its confirmation on biopsy and tzank smear and clinical examination of all mucosal surfaces to establish prevalence and pattern of eye involvement in the patients of pemphigus vulgaris. Eye involvement was found in $32(32 \%)$ patients of which the most common symptom was found to be conjunctival redness which was found in 18 patients $(18 \%)$. Eyelid skin involvement was also quite common in 17 patients (17\%). Conjunctival discharge was found in $15 \%$ of patients. Blurring of vision was found in only $4(4 \%)$ of patients. Pain was quite common and noted in about 21 patients (21\%).Eye involvement was found bilaterally in 27 patients ( $90 \%$ of patients with eye involvement) with 3 patients having unilateral eye involvement. Photophobia was present in 7 (7\%) patients. Bulbar conjunctival erosions were found in 2 patients $(2 \%)$. These all discussed problems were present during the course of pemphigus vulgaris and not present before or during the remission of the disease. These eye problems gradually got improved as systemic disease got improved during the treatment with systemic corticosteroids, immunosuppressants, local symptomatic treatment and eye care (Table 1). 
Table I Signs \& Symptoms of Eye Involvement

\begin{tabular}{lll}
\hline Eyes Signs and symptoms & Patients involved & Patients \%age involved \\
\hline Conjunctival Redness & 18 & $18 \%$ \\
Conjunctival Discharge & 15 & $15 \%$ \\
Photophobia & 7 & $07 \%$ \\
Pain/Burning/Stinging & 21 & $21 \%$ \\
Blurring of vision & 4 & $04 \%$ \\
Eyelid skin Erosions & 17 & $17 \%$ \\
Conjunctival Erosions & 2 & $02 \%$ \\
Foreign Body Sensation & 12 & $12 \%$ \\
\hline
\end{tabular}

The sum of percentages of individual eye manifestations is not equal to $32 \%$ because many of our patients of pemphigus vulgaris with eye involvement showed multiple manifestations.

\section{Discussion}

Nearly all patients with pemphigus have mucosal lesions and oral lesions were found in $50-70 \%$ of patients. Other mucosal surfaces may be involved including conjunctiva, nasopharynx, larynx, oesophagus, urethra, vulva and cervix but there statistics are not well defined. We studied 100 pemphigus vulgaris patients admitted in dermatology department of Mayo hospital during last 2 years clinically to study the trends of eye involvement in our population (Asian population) with pemphigus vulgaris. In our study, eye involvement was seen in $32 \%$ of the patients while study held in Spanish population from 1985 to $2014^{8}$ showed $14 \%$ eye involvement and it showed that in our population eye involvement was more than 2 times of Spanish population. ${ }^{8}$ In our study the most common presentation was conjunctival redness which was found in 18 patients (18\%) and least common presentation of conjunctival involvement was conjunctival erosions which were seen in 2 patients $(2 \%)$. There was bilateral blurring of vision seen as possible involvement of cornea in only 4 patients (4\%).Our study concluded that conjunctival lesions are more common in our population. The ocular lesions responded well to the local eye care and systemic treatment for pemphigusvulgaris.

Ocular involvement in PV has been reported but its prevalence and clinical characteristics are not well defined. Akhyani $\mathrm{M}$ et al. ${ }^{9}$ conducted a prospective study to know the pattern and frequency of eye involvement in lranian population. That study showed a significant percentage of patients with eye involvement and the most common manifestation was conjunctivitis. So conjunctivitis was the most observed type of manifestation followed by erosion of the palpebral conjunctiva as studied by Akhyani $\mathrm{M}$ et al. ${ }^{9}$ Erosion of the bulbar conjunctiva was noted in only one patient of sample population. No significant relation was found between ocular involvement and disease activity (partial remission or relapse). The conclusion of study by Akhyani $\mathrm{M}$ et al. ${ }^{9}$ was that ocular involvement is not rare in PV; $16.5 \%$ of PV patients develop ocular disease independent of the disease activity and extension. Conjunctivitis is the most common type of involvement, however, palpebral conjunctival erosion is more frequent than previously realized and in our study we also concluded that eye involvement is common asit was seen in $32 \%$ of patients in the admitted patients of pemphigus vulgaris and most common pattern of ocular involvement is conjunctival mucosal involvement showing the symptoms of conjunctivitis and severe eye involvement is rather a rare presentation as concluded by blurring of vision and conjunctival erosions are rare presentation in our study and the studies compared with our study. Corneal involvement was not a presentation with pemphigus vulgaris and it was the conjunctiva that was involved with the disease. ${ }^{10-14}$ According to a study by D Temilola in pemphigus vulgaris patients, eye involvement was seen and it was mostly inflammation of conjunctiva that caused burning sensation in eyes and production of tears causing watery discharge..$^{15}$

According to another study by Baykal HE, the eye involvement was not as much common but the most common manifestation was conjunctivitis. ${ }^{16}$ According to a study held by Lifshitz T et al. ${ }^{17}$ although ocular involvement in pemphigus was not as common but the pattern of manifestation was same and conjunctivitis was the most commonly seen manifestation even in this study. ${ }^{17}$

\section{Conclusion}

As concluded from our study, ocular involvement is quite commonly associated in pemphigus vulgaris patients so careful eye clinical examination and subsequent treatment of patient with proper care can reduce morbidity of the pemphigus vulgaris patients with eye involvement.

\section{Acknowledgment}

None.

\section{Funding}

None.

\section{Conflicts of interest}

The authors report no conflicts of interest.

\section{References}

1. Nousari HC, Anhalt GJ. Pemphigus \& bullous pemphigoid. The Lancet 1999;354:667-672.

2. Mahoney MG, Wang Z, Rothenberger K, et al. Explanations for the clinical and microscopic localization of lesions in pemphigus foliaceus and vulgaris. J Clin Invest.1999;103(4):461-468.

3. Seishima M, Oyama Z, Shimizu H, et al. Pemphigus of the eyelids. Eur $J$ Dermatol. 2001;11(2):141-143. 
4. Ekong AS, Foster CS, Roque MR. Eye involvement in autoimmune blistering diseases. Clin Dermatol. 2001;19(6):742-749.

5. Hodak E, Kremer I, David M, et al. Conjunctival involvement in pemphigus vulgaris: a clinical, histopathological and immunofluorescence study. Br J Dermatol. 1990;123(5):615-620.

6. Destro M, Wallow IL, Brightbill FS. Recessive dystrophic epidermolysis bullosa. Arch Ophthalmol. 1987;105(9):1248-52.

7. Figueira EC, Murrell DF, Coroneo MT. Ophthalmic involvement in inherited epidermolysis bullosa. Dermatol Clin. 2010;28(1):143-152.

8. Espana A, Iranzo P, Herrero GJ, et al. Ocular involvement in pemphigus vulgaris-a retrospective study of a large Spanish cohort. J Dtsch Dermatol Ges. 2017;15(4):396-403.

9. Akhyani M, Keshtkar JA, Chams DC, et al. Ocular involvement in pemphigus vulgaris. J Dermatol. 2014;41(7):618-621.

10. Uludag HA, Uysal Y, Kucukevcilioglu M, et al. An uncommon ocular manifestation of pemphigus vul-garis: conjunctival mass. Ocular Immunology and Inflammation. 2013;21(5):400-402.
11. Palleschi GM, Giomi B, Fabbri P. Ocular involvement in pemphigus. Am J Ophthalmol. 2007;144(1):149-152.

12. Merchant S, Weinstein M. Pemphigus vulgaris: the eyes have it. Pediatrics. 2003;112(1):183-185.

13. Olszewska M, Komor M, Mazur M, et al. Response of ocular pemphigus vulgaris to therapy. Case report and review of literature. J Dermatol Case Rep. 2008;2(1):1-3.

14. De Souza RS, Da Silva JB, dos Santos CC, et al. Manifestation of vulgar pemphigus in eyelid mucosa: clinical case report. Journal of Ophthalmology and Visual Neurosciences. 2017;2:1-4.

15. Temilola D, Holmes H. Oral pemphigus vulgaris with skin and ocular involvement. J Dent South A .2018;73(2):83-85.

16. Baykal HE, Pleyer U, Sonnichsen K, et al. Severe eye involvement in pemphigus vulgaris. Ophthalmologe. 1995;92(6):854-857.

17. Lifshitz T, Levy J, Cagnano E, et al. Severe conjunctival and eyelid involvement in pemphigus vulgaris. Int Ophthalmol. 2004 ;25(2):73-74. 